\title{
First detection of oscillations in the Halo giant HD 122563: Validation of seismic scaling relations and new parameters ${ }^{\star}$
}

\author{
O. Creevey ${ }^{1}$, F. Grundahl ${ }^{2}$, F. Thévenin ${ }^{1}$, E. Corsaro ${ }^{3}$, P. L. Pallé ${ }^{4,5}$, D. Salabert ${ }^{6,7, \star \star}$, B. Pichon ${ }^{1}$, R. Collet ${ }^{2}$, \\ L. Bigot ${ }^{1}$, V. Antoci ${ }^{2}$, and M. F. Andersen ${ }^{2}$ \\ 1 Université Côte d'Azur, Observatoire de la Côte d'Azur, CNRS, Laboratoire Lagrange, France \\ e-mail: orlagh.creevey@oca.eu \\ 2 Stellar Astrophysics Centre, Department of Physics and Astronomy, Aarhus University, Ny Munkegade 120, 8000 Aarhus C, \\ Denmark \\ 3 INAF - Osservatorio Astrofisico di Catania, via S. Sofia 78, 95123 Catania, Italy \\ ${ }^{4}$ Instituto de Astrofisica de Canarias, 38205 La Laguna, Tenerife, Spain \\ 5 Universidad de La Laguna (ULL), Departamento de Astrofisica, 38206 La Laguna, Tenerife, Spain \\ ${ }^{6}$ IRFU, CEA, Université Paris-Saclay, 91191 Gif-sur-Yvette, France \\ 7 Université Paris Diderot, AIM, Sorbonne Paris Cité, CEA, CNRS, 91191 Gif-sur-Yvette, France
}

Received 26 November 2018 / Accepted 4 February 2019

\begin{abstract}
Aims. The nearby metal-poor giant HD 122563 is an important astrophysical laboratory in which to test stellar atmospheric and interior physics. It is also a benchmark star for which to calibrate methods to apply to large scale surveys. Recently it has been remeasured using various methodologies given the new high precision instruments at our disposal. However, inconsistencies in the observations and models have been found.

Methods. In order to better characterise this star using complementary techniques we have been measuring its radial velocities since 2016 using the Hertzsprung telescope (SONG network node) in order to detect oscillations.

Results. In this work we report the first detections of sun-like oscillations in this star, and to our knowledge, a detection in the most metal-poor giant to date. We applied the classical seismic scaling relation to derive a new surface gravity for HD 122563 of $\log g_{v}=1.39 \pm 0.01$. Reasonable constraints on the mass imposed by its PopII giant classification then yields a radius of $30.8 \pm 1.0 \mathcal{R}_{\odot}$. By coupling this new radius with recent interferometric measurements we infer a distance to the star of $306 \pm 9$ pc. This result places it further away than was previously thought and is inconsistent with the HIPPARcos parallax. Independent data from the Gaia mission corroborate the distance hypothesis $\left(d_{\mathrm{GDR} 2}=290 \pm 5 \mathrm{pc}\right)$, and thus the updated fundamental parameters.

Conclusions. We confirm the validity of the classical seismic scaling relation for surface gravity in metal-poor and evolved star regimes. The remaining discrepancy of 0.04 dex between $\log g_{\mathrm{GDR} 2}(=1.43 \pm 0.03)$ reduces to 0.02 dex by applying corrections to the scaling relations based on the mean molecular weight and adiabatic exponent. The new constraints on the Hertzsprung-Russell diagram $\left(L_{\star v}=381 \pm 26 \mathcal{L}_{\odot}\right)$ significantly reduce the disagreement between the stellar parameters and evolution models, however, a discrepancy of the order of $150 \mathrm{~K}$ still exists. Fine-tuned stellar evolution calculations show that this discrepancy can be reconciled by changing the mixing-length parameter by an amount $(-0.35)$ that is in agreement with predictions from recent 3D simulations and empirical results. Asteroseismic measurements are continuing, and analysis of the full frequency data complemented by a distance estimate promises to bring important constraints on our understanding of this star and of the accurate calibration of the seismic scaling relations in this regime.
\end{abstract}

Key words. asteroseismology - methods: observational - stars: fundamental parameters - stars: individual: HD 122563 stars: distances - stars: Population II

\section{Introduction}

HD $122563\left(V=6.2 \mathrm{mag}, 14^{\mathrm{h}} 02^{\mathrm{m}} 31.8^{\mathrm{s}},+09^{\circ} 41^{\prime} 09.95^{\prime \prime}\right)$ is one of the brightest and closest metal-poor $[\mathrm{M} / \mathrm{H}]=-2.4$ (Collet et al. 2018; Prakapavičius et al. 2017) giant stars that offers the unique opportunity to be observed and analysed using many different methodologies. There are few stars for which such a complete set of observations can be obtained. The advantage of this is that we can obtain robust (almost) model-independent determinations of many of its fundamental

* SONG radial velocities are only available at the CDS via anonymous ftp to cdsarc.u-strasbg.fr (130.79.128.5) or via http: //cdsarc.u-strasbg.fr/viz-bin/qcat?]/A+A/625/A33

$\star \star$ Previous affiliation. parameters, but many of these can also be compared using different methodologies, and so it allows us to investigate sources of systematic errors. For example, it serves as a benchmark star for testing stellar astrophysics, such as non-local thermodynamical equilibrium (NLTE) effects in stellar atmospheres (Heiter et al. 2015; Thévenin \& Idiart 1999; Collet et al. 2005), or 3D stellar atmosphere structure (Prakapavičius et al. 2017; Collet et al. 2018). In stellar evolution models strong assumptions on its age, mass and initial helium abundance can be made which allows one to investigate tunable parameters or details of the interior physics. HD 122563 also contributes to calibrating large Galactic surveys, for example, Gilmore et al. (2012), which aim to understand the evolution of the Milky Way (Jofré et al. 2017). 
In Creevey et al. (2012b), from hereon C12, strong constraints are placed on the position of this star in the HertzsprungRussell (HR) diagram, in particular with the determination of its interferometric diameter. These authors find that classical stellar evolution models are unable to reproduce its position within the error box. One of the proposed solutions by these authors was to change the mixing-length parameter $\alpha$ in the stellar evolution codes, and they managed to produce a model which correctly fit the observational data. Such changes are not unrealistic (e.g. Bonaca et al. 2012; Creevey et al. 2017; Tayar et al. 2017; Joyce \& Chaboyer 2018; Viani et al. 2018), however, the size of the modification suggested that something should be addressed either in the models for very metal-poor stars or in the observations. Even with such constraints on the model parameters, its derived age is not well constrained, due to degeneracies between the unobservable mass and initial helium mass fraction, see Lebreton et al. (1999) for a discussion. In C12 it is proposed that asteroseismic observations could help to constrain these other parameters, which would improve the age determination and perhaps shed some light on the difficulty of matching the HR diagram constraints with classical models.

HD 122563 has also been the subject of several recent atmospheric studies (Amarsi et al. 2018), and some discrepancies in analyses were noted, for example, the 3D non-LTE analysis of hydrogen lines shows a spread of $T_{\text {eff }}$ values depending on the line used. Collet et al. (2018) suggest that a lowering of the surface gravity from their adopted $\log g=1.61 \pm 0.07$ would reduce the oxygen abundance discrepancy between molecular and atomic species. In $\mathrm{C} 12$ the authors also predict a value $\log g=1.60 \pm 0.04$ based on the measured diameter, available parallax, and a model-constrained mass.

Given the current questions and indications that the models or the observations are not entirely consistent, we proposed to observe the star in multi-epoch spectrography in order to detect stellar oscillations. Asteroseismic analysis would provide a fresh new perspective, and hopefully help to unravel the current inconsistencies, while also allowing us to investigate the scaling relation in the non-solar regime. In this paper we report the first detection of stellar oscillations in HD 122563 using the Hertzsprung SONG telescope located on the Observatorio del Teide. This is the most metal-poor star (to our knowledge) to have sun-like oscillations detected ${ }^{1}$. We measured the global seismic quantity $v_{\max }$ for HD 122563 (Sect. 2) and along with complementary information we derive a new surface gravity, radius and distance to the star (Sect. 3). New data from the Gaia mission (Gaia Collaboration 2018) corroborate our results. We then discuss the implications of our results on the seismic scaling relation for $\log g$, the position of the star in the HR diagram and 1D stellar models (Sects. 4 and 5).

\section{Observations of HD 122563}

\subsection{New asteroseismic observations from radial velocities}

We obtained time series radial velocity observations with the $1 \mathrm{~m}$ Hertzsprung SONG telescope equipped with an echelle spectrograph and located at the Observatorio del Teide. The Hertzsprung telescope is a node of the Stellar Observations Network Group (SONG). From April 2016 to July 2017 we obtained an average of one spectrum per night when the object is visible. The spectra were reduced and calibrated using the SONG pipeline. Details of the Hertzsprung telescope characteristics

\footnotetext{
1 Epstein et al. (2014) report a list of nine metal-poor stars with
} detected oscillations with abundances larger than -2.2 dex.

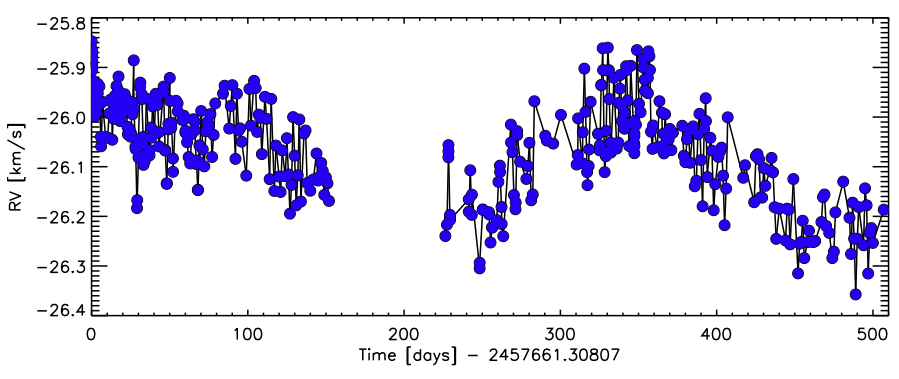

Fig. 1. Radial velocity time series of HD 122563. The oscillations are on the timescale of a few days, and a long-term trend of the order of 300 days is visible. See Sect. 2.1.1.

and reduction pipeline are given in Andersen et al. (2014) and Grundahl et al. (2017). All observations were obtained using an iodine cell for precise wavelength calibration. A spectral resolution of 80000 and an exposure time of 900s was used throughout.

The radial velocity (RV) time series is presented in Fig. 1. It comprises 387 data points over a total of 449 nights. The typical uncertainty on the RV was found to be in the $11-14 \mathrm{~m} \mathrm{~s}^{-1}$ range, depending on the signal-to-noise ratio in the observed spectrum. However, as can be seen from the figure, there is a long-term trend. We believe that this could be evidence of convection, rotation or activity. As the trend is of the order of 300 days, far from the expected intrinsic pulsation periods, we performed a frequency analysis directly on the time series produced by the pipeline.

\subsubsection{Time-series analysis}

The power spectrum of the velocity time-series was initially calculated as an unweighted least-squares fit of sinusoids (Frandsen et al. 1995; Arentoft et al. 1998; Bedding et al. 2004; Kjeldsen et al. 2005; Corsaro et al. 2012), and converted into power spectral density (PSD) by normalising for the spectral resolution, namely the integral of the spectral window, of $\sim 0.06 \mu \mathrm{Hz}$. We also tested the case of a weighted least-squares fit to check for possible improvements in the signal-to-noise. For this purpose we used a weight assigned to each point of the radial velocity time-series according to the corresponding uncertainty estimate obtained from the SONG pipeline ${ }^{2}$. The radial velocity uncertainties were previously rescaled in order to correct for the presence of possible outliers, following the approach presented by Butler et al. (2004; see also Corsaro et al. 2012). Finally we measured the amplitude of the noise level in the amplitude spectrum, in the region $4-6 \mu \mathrm{Hz}$, outside the power excess due to oscillations, for both the weighted and unweighted case. We find that the amplitude of the noise is lower in the unweighted case, reaching down to $11.8 \mathrm{~m} \mathrm{~s}^{-1}$. We therefore decided to adopt the un-weighted spectrum for further analysis.

\subsubsection{Extraction of global seismic parameters $v_{\max }$}

We used the Diamonds Bayesian Inference tool (Corsaro \& De Ridder 2014, Appendix A) to model the power spectral density (PSD) of the star. The PSD and the best-fit model are shown in Fig. 2, and incorporates a flat noise component, two Harvey-like profiles to account for granulation-driven signal,

2 It is noted in the SONG documentation that the uncertainties reported on the RV data should be considered with caution. 


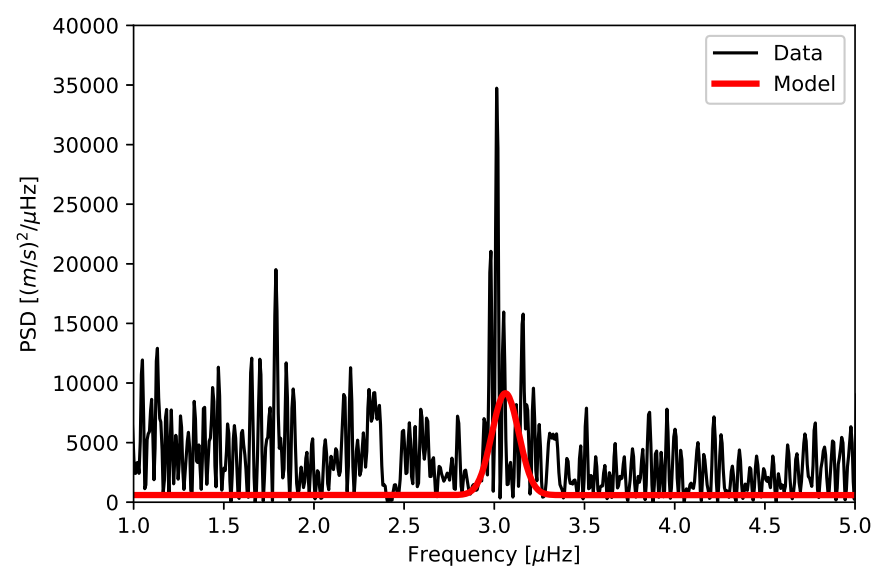

Fig. 2. Power spectral density and background model fit with Diamonds to determine $v_{\max }$. The red line shows the total fit, including the oscillation power excess. See Sect. 2.1.2.

and a Gaussian envelope to model the oscillation power excess (Corsaro et al. 2015). A clear excess of power due to the oscillations is detected at $3 \mu \mathrm{Hz}$ (see Table 1), this is referred to as $v_{\max }$. We note that the width of the power excess is narrow, and this seems to follow the trend presented by Yu et al. (2018), although their sample only goes as low as $40 \mu \mathrm{Hz}$. The results reported in Table 1 are obtained from calculating the 16, 50, and 84 percentiles of the marginalised distribution of $v_{\max }$. We note that lower and upper confidence intervals are strictly formal uncertainties, without consideration of possible systematic errors. As possible sources of errors, we also determined $v_{\max } 1$ ) after filtering for the low-frequency signal, and 2) using a flat background in the power spectrum. In both cases our results are consistent with those reported in Table 1.

\subsection{Literature values of the effective temperature of HD 122563}

The $T_{\text {eff }}$ of HD 122563 has been derived using many independent methods, with all methods showing agreement within a range of $\pm 100 \mathrm{~K}$ around $4600 \mathrm{~K}$. A recent compilation of spectroscopically derived $T_{\text {eff }}$ is given in Heiter et al. (2015) who recommend $4587 \pm 60 \mathrm{~K}$. Casagrande et al. (2014) used the (quasi-) model independent infra red flux method (IRFM) and derived a $T_{\text {eff }}=4600 \pm 47 \mathrm{~K}$. Two recent analyses by C12 and Karovicova et al. (2018, K18 hereafter) using interferometry obtain results also in agreement, $4598 \pm 41 \mathrm{~K}$ and $4636 \pm 36 \mathrm{~K}$. These values are obtained using independent determinations of the angular diameter $\theta$ and the bolometric flux $F_{\text {bol }}$ (Table 1). For the latter both adapt a value of extinction ${ }^{3}$ of $A_{V}=$ $0.01 \mathrm{mag}$. The agreement between all of the determinations provides good confidence in the $T_{\text {eff }}$ and the assumed low value of extinction.

In this work we do not rederive $T_{\text {eff }}$ but we adopted the two independent interferometric determinations from $\mathrm{C} 12$ and K18. However, we used their reported bolometric flux $F_{\text {bol }}$ and measured angular diameter $\theta$ in order to correctly propagate the uncertainties on all of our inferences. Furthermore, $\theta$ is used

\footnotetext{
3 In this work we rederived $F_{\text {bol }}$ using the iterative method described in $\mathrm{C} 12$, but adopting the bolometric corrections from Houdashelt et al. (2000). This was done with the aim of exploring the effect of a nonneglible interstellar extinction (Sect. 4). The new $F_{\text {bol }}$ change slightly which result in a $T_{\text {eff }}=4610 / 4629 \mathrm{~K}$ for C12/K18 for $A_{V}=0.01 \mathrm{mag}$.
}

Table 1. Observed properties of HD 122563 used in this work.

\begin{tabular}{lccc}
\hline \hline Property & & Value & Source \\
\hline$v_{\max }$ & $(\mu \mathrm{Hz})$ & $3.07_{-0.04}^{+0.05}$ & This work \\
$\theta_{\mathrm{LD}, \mathrm{A}}$ & $(\mathrm{mas})$ & $0.940 \pm 0.011$ & $\mathrm{C} 12$ \\
$F_{\mathrm{bol}, \mathrm{A}}$ & $\left(\mathrm{erg}^{-1} \mathrm{~s}^{-1} \mathrm{~cm}^{-2}\right)$ & $13.16 \pm 0.36 \mathrm{e}-8$ & $\mathrm{C} 12$ \\
$T_{\text {eff,A }}$ & $(\mathrm{K})$ & $4598 \pm 41$ & $\mathrm{C} 12$ \\
$\theta_{\mathrm{LD}, \mathrm{B}}$ & $(\mathrm{mas})$ & $0.926 \pm 0.011$ & $\mathrm{~K} 18$ \\
$F_{\mathrm{bol}, \mathrm{B}}$ & $\left(\mathrm{erg}^{-1} \mathrm{~s}^{-1} \mathrm{~cm}^{-2}\right)$ & $13.20 \pm 0.29 \mathrm{e}-8$ & $\mathrm{~K} 18$ \\
$T_{\text {eff, }}$ & $(\mathrm{K})$ & $4636 \pm 36$ & $\mathrm{~K} 18$ \\
$\pi_{\mathrm{HIPP}}$ & $(\mathrm{mas})$ & $4.22 \pm 0.36$ & van Leeuwen $(2007)$ \\
$\pi_{\mathrm{GDR} 2}$ & $(\mathrm{mas})$ & $3.444 \pm 0.063$ & Gaia Collaboration $(2018)$ \\
\hline
\end{tabular}

along with a parallax to perform a similar exercise in order to compare our results (see Sect. 3.2). By adopting both $\mathrm{C} 12$ and $\mathrm{K} 18$ one can investigate the effect of a possible source of systematic error from $\theta$. Considering an extreme value of extinction we investigated a source of error in $F_{\text {bol }}$, and consequently its $T_{\text {eff }}$ (Sect. 4).

\section{Surface gravity and distance of HD 122563}

\subsection{Surface gravity and distance from asteroseismic observations}

It has been demonstrated in many papers that the surface gravity of a star can be derived with very high precision using asteroseismic observations. For example, Brown \& Gilliland (1994), Chaplin et al. (2011), Creevey et al. (2012a). Even using simple scaling relations, such as that proposed by Kjeldsen \& Bedding (1995, KB95 hereafter), $\log g$ can be easily derived. Direct comparisons between $\log g$ derived from seismology and from other methods have also demonstrated its accuracy (Morel \& Miglio 2012; Hekker et al. 2013). For non-evolved stars typical errors, including systematics on the input parameters and accuracy, are of the order of 0.04 dex (Creevey et al. 2013). However, these scaling relations are based on the Sun, and as the star begins to differ from the Sun, the relations may begin to deviate from solar-scaled values. Several authors have addressed this issue recently (Hekker et al. 2013; Coelho et al. 2015; Sharma et al. 2016; Viani et al. 2017; Kallinger et al. 2018; Brogaard et al. 2018) and propose modifications to the scaling relations.

The classical relation for the asteroseismic quantity $v_{\max }$ is

$\frac{v_{\max }}{v_{\max \odot}}=f_{v_{\max }} \frac{g}{g_{\odot}} \sqrt{\frac{T_{\mathrm{eff} \odot}}{T_{\mathrm{eff}}}}$,

where $f_{v_{\max }}=1.0, v_{\max , \odot}=3050 \mu \mathrm{Hz}$ and $T_{\text {eff }, \odot}=5777 \mathrm{~K}$ (Kjeldsen \& Bedding 1995). In this work we have adopted $\log g_{\odot}=4.438$ dex from the IAU convention 4 (Prša et al. 2016), and thus we consequently adopt $T_{\text {eff, } \odot}=5772 \mathrm{~K}$. Revised relations are presented in some of the references cited above where $f_{v_{\max }} \neq 1.00$, or $v_{\max , \odot} \neq 3050 \mu \mathrm{Hz}$, and these are both discussed below.

Recent analysis by Viani et al. (2017, V17 hereafter) replaces $f_{v_{\max }}$ explicitly with terms associated with the mean molecular weight and the adiabatic exponent, the theoretical basis for which has been studied in, for example Belkacem et al. (2011). However, as these terms can not be derived without a stellar model, we are interested in testing the classical relation, but we

\footnotetext{
4 https://wWw.iau.org/static/resolutions/IAU2015_ English.pdf; Resolution B3
} 


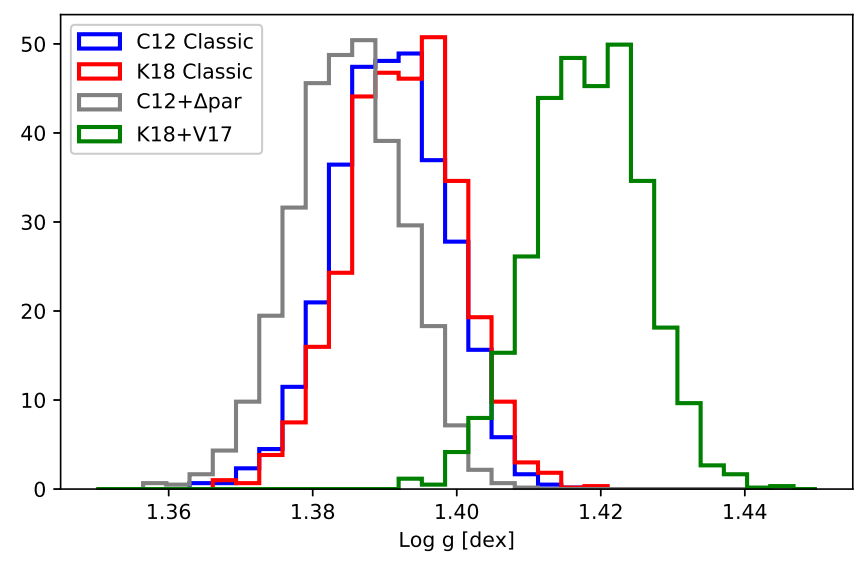

Fig. 3. Distributions of $\log g$ derived from asteroseismic data using the seismic scaling relation. The blue and red represent the results using the observed properties from $\mathrm{C} 12$ and $\mathrm{K} 18$, respectively, and $f_{v_{\max }}=1.0$. The grey and green lines show $\log g$ using the different solar reference values and a revised scaling relation (Viani et al. 2017), respectively. See Sect. 3.1 for details.

do consider both cases. We also note that while these corrections can be important, they do not fully account for the effects of changes in composition on the atmospheric opacities and on the structure of the outer layers (as acknowledged by the authors). There are important deviations between 1D and 3D stellar models in terms of the surface stratifications at very low metallicity regimes, and differences between $1 \mathrm{D}$ and $3 \mathrm{D}$ stratifications contribute to this surface effect (Trampedach et al. 2017).

Using Eq. (1) in its classical form, we calculate the surface gravity of HD 122563 from $v_{\max }$ and $T_{\text {eff. }}$ To correctly propagate the uncertainties reported in $\mathrm{C} 12$ and $\mathrm{K} 18$, and the determination of $v_{\max }$, we performed Monte-Carlo-like simulations to derive $\log g$. More specifically for each simulation we perturb the observed quantity $\left(F_{\text {bol }}, \theta\right)$ by adding a random number drawn from a normal distribution with width 1 scaled by its symmetric uncertainty. For $v_{\max }$ we used the marginal distribution directly from Sect. 2. The distributions of the resulting values of $\log g$ from the simulations are shown in Fig. 3 adopting the two referenced interferometric measurements (blue and red, respectively). The value of $\log g_{v}$ for K18 (red) is $1.393 \pm 0.007$ dex, where the sub-script $v$ denotes an asteroseismically-derived value. The median values using $\mathrm{C} 12$ and $\mathrm{K} 18$ differ by 0.0018 dex. A possible source of systematic error arises from the definition of the

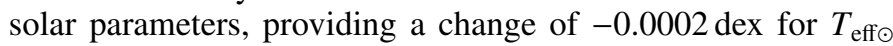
and -0.0056 for $v_{\max \odot}$, and the combined change is shown for $\mathrm{C} 12$ by the grey histogram.

For the more recent investigations of the scaling relation, $f_{v_{\max }}$ is replaced by terms associated with the mean molecular weight $\left(\mu / \mu_{\odot}\right)^{1 / 2}$ or a combination of $\mu$ and the adiabatic exponent $\left(\Gamma_{1} / \Gamma_{1 \odot}\right)^{1 / 2}$. This is justified by the fact that $v_{\max }$ is expected to scale with the cut-off frequency in the atmosphere (see references above). In practice, these values are not readily obtained and require the use of stellar models. For $\mu$ this may be estimated by making assumptions about the helium abundance of the star and using the observed metallicity. For $\Gamma_{1}$ a stellar model is needed. Using the stellar models from Sect. 4 we calculated $\mu_{\star}=0.5904$ and $\Gamma_{1 \star}=1.545$, and adopted the solar values, $\mu_{\odot}=0.6159$ and $\Gamma_{1 \odot}=5 / 3$, in order to derive the corrections to the scaling relation.

Applying the correction associated only with $\mu$ results in an increased $\log g$ of 0.007 dex. However, applying the correction associated with $\Gamma_{1}$ has a more significant impact. The resulting

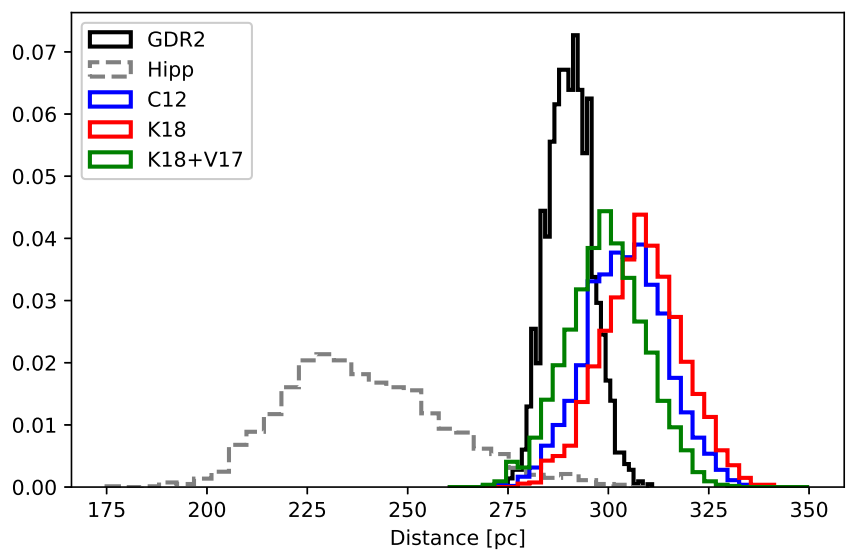

Fig. 4. Distances to HD 122563 derived using parallaxes (black and grey) and asteroseismic inferences (blue, red and green).

distribution for K18 is represented by the green histogram in Fig. 3. It results in a systematic change of +0.0256 dex to yield a $\log g_{v}=1.418 \pm 0.007$ (see Table 2).

Given cosmological constraints (upper age) and our knowledge of stellar evolution, we can assume that the mass of this evolved star is likely between 0.80 and $0.90 \mathcal{M}_{\odot}(\mathrm{C} 12)$. By adopting a conservative prior on the mass of $0.85 \pm 0.05 \mathcal{M}_{\odot}$, the radius $R$ can be inferred from $\log g$ and the mass prior. This then gives us access to the distance of the star, because the angular diameter has been measured. From the classical relation with $f_{v_{\max }}=1.0 \mathrm{we}$ derive a radius of $30.8 \pm 1.0 \mathcal{R}_{\odot}$ implying a distance of $305 \pm 10 \mathrm{pc}$ for C12 (see Table 2). The smallest derived distance is $296 \pm 9$ pc using $\mathrm{C} 12+\mathrm{V} 17$. These distances are larger than that proposed by van Leeuwen (2007) who measures its parallax of $4.22 \pm 0.35$ mas using data from the HiPPARcos mission. The derived distances are illustrated in Fig. 4 using the same colour-coding as in Fig. 3, with the latter denoted by the grey dashed lines.

\subsection{Surface gravity and distance from Gaia DR2 measurements}

The Gaia DR2 catalogue (Gaia Collaboration 2018; Lindegren et al. 2018) provides a new and more precise parallax for HD 122563 of $3.444 \pm 0.063$ mas. The distance to the star inferred from this newer parallax is then $290 \pm 5 \mathrm{pc}$ if we assume no prior, and thus $30 \%$ further away than previously thought (see Fig. 4, black distribution). This value is consistent at the $1 \sigma$ level with the values obtained using asteroseismology. Following the methodology from Sect. 3.1, using the angular diameter and the parallax measurement and adopting a mass prior, we infer (a new radius) and $\log g$ for HD 122563. This results in $\log g_{\mathrm{GDR} 2}=1.432_{-0.033}^{+0.030}$ and $1.445_{-0.033}^{+0.031}$ using $\mathrm{C} 12$ and $\mathrm{K} 18$, respectively. We furthermore used $F_{\text {bol }}$ to derive luminosity $L_{\star}$ (Sect. 4). The distance $d$, radius, surface gravity and $L_{\star}$ are summarised in Table 2 under the heading "GDR2 Parallax".

A potential systematic error of +0.029 mas on the parallax has been documented in the Gaia Second Data Release (Luri et al. 2018). This zero point corresponds to the difference between the median value of the quasars observed by Gaia, which are assumed to have no parallactic motion, and zero. For completeness, we apply this error also in our analysis, and calculate the corresponding parameters (Table 2). A larger parallax implies a smaller distance and radius, and a higher value of $\log g$. This is further away from the seismic value. However, as this zeropoint is a value derived for faint quasars, there is no reason to expect it to apply to the brighter end of the Gaia spectrum. 
Table 2. Derived properties of HD 122563 using asteroseismology (left) and Gaia parallaxes (right).

\begin{tabular}{lccccccccc}
\hline \hline & & \multicolumn{4}{c}{ Seismology } & \multicolumn{3}{c}{ GDR2 parallax } \\
\cline { 3 - 10 } & & \multicolumn{2}{c}{ KB95 } & \multicolumn{2}{c}{ V17 } & \multicolumn{2}{c}{$\mathrm{s}(\pi)=0.0$} & \multicolumn{2}{c}{$\mathrm{s}(\boldsymbol{\pi}) \neq 0.0$} \\
\cline { 3 - 10 } & & $\mathrm{C} 12$ & $\mathrm{~K} 18$ & $\mathrm{C} 12$ & $\mathrm{~K} 18$ & $\mathrm{C} 12$ & $\mathrm{~K} 18$ & $\mathrm{C} 12$ & $\mathrm{~K} 18$ \\
\hline $\log g$ & $(\mathrm{dex})$ & $1.391_{-7}^{+8}$ & $1.393_{-7}^{+7}$ & $1.416_{-7}^{+8}$ & $1.418_{-7}^{+7}$ & $1.433_{-32}^{+30}$ & $1.446_{-32}^{+30}$ & $1.441_{-33}^{+30}$ & $1.453_{-33}^{+31}$ \\
$d$ & $(\mathrm{pc})$ & $305_{-10}^{+10}$ & $308_{-10}^{+10}$ & $296_{-9}^{+9}$ & $299_{-10}^{+10}$ & $290_{-5}^{+5}$ & - & $288_{-5}^{+5}$ & - \\
$R_{\star}$ & $\left(\mathcal{R}_{\odot}\right)$ & $30.8_{-10}^{+10}$ & $30.7_{-9}^{+9}$ & $29.9_{-9}^{+9}$ & $29.8_{-9}^{+9}$ & $29.4_{-7}^{+6}$ & $28.9_{-6}^{+7}$ & $29.1_{-6}^{+7}$ & $29.7_{-6}^{+6}$ \\
$L_{\star}$ & $\left(\mathcal{L}_{\odot}\right)$ & $381_{-26}^{+26}$ & $392_{-26}^{+27}$ & $359_{-24}^{+25}$ & $370_{-24}^{+25}$ & $346_{-15}^{+16}$ & $347_{-15}^{+15}$ & $340_{-16}^{+15}$ & $341_{-14}^{+14}$ \\
\hline
\end{tabular}

Notes. For the seismology parameters, we show the results using the classic scaling relation (KB95) and that given in V17, using both C12 and K18 measurements. For the results using the GDR2 parallax we show the results without and with a systematic error $s(\pi)$ of +0.029 mas on the parallax, and again for $\mathrm{C} 12$ and K18.

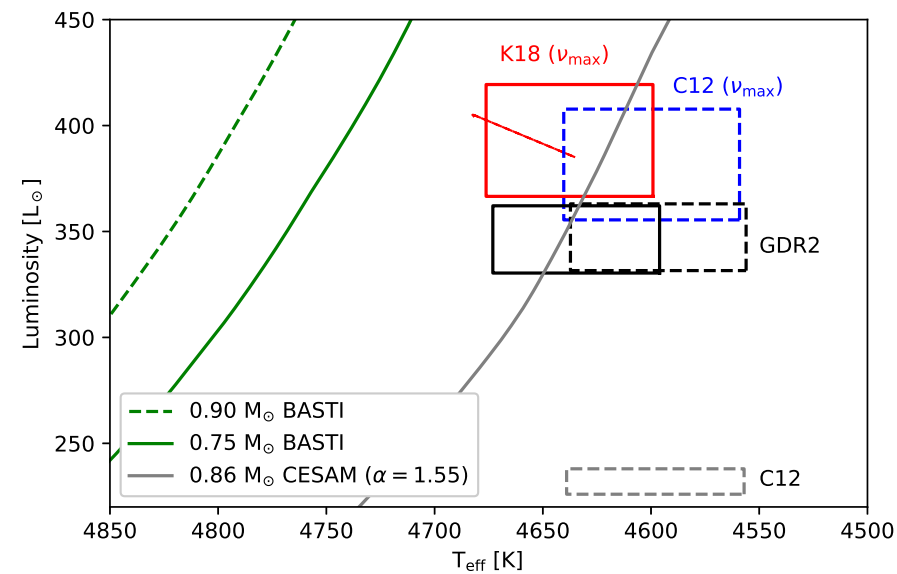

Fig. 5. HR diagram presenting the revised positions of HD 122563 based on asteroseismic data (red and blue) and Gaia data (black) for $\mathrm{K} 18$ and $\mathrm{C} 12$ (continuous and dashed lines, respectively). The value presented in C12 using the parallax from van Leeuwen (2007) is shown with the grey dashed box. The red vector represents a potential shift in the median values of $L_{\star}$ and $T_{\text {eff }}$ if we consider an extinction $A_{V}=$ $0.08 \mathrm{mag}$ (the reference value is $0.01 \mathrm{mag}$ ). Classical evolution tracks for a 0.75 and $0.90 \mathcal{M}_{\odot}$ model from BASTI (Pietrinferni et al. 2004) are shown by the green lines, while a fine-tuned $0.86 \mathcal{M}_{\odot}$ model using the CESAM2K evolution code (Morel 1997) with a reduced value of the mixing-length parameter compared to the solar one is shown by the grey continuous curve. See Sect. 4 for details.

\section{Observational constraints in the HR diagram}

We plot the position of the new observational constraints in the HR diagram in Fig. 5 using the results from the classical scaling relation and considering no systematic error on the parallax. We also show BASTI evolutionary tracks (Pietrinferni et al. 2004) for a 0.75 and $0.90 \mathcal{M}_{\odot}$ star using standard solar scaled physics (non-canonical, alpha-enhanced shifts the tracks to hotter temperatures). These tracks are frequently used in the literature. The new HR diagram constraints are shown using the same colourcode as Fig. 3 with the grey dashed lines representing C12 constraints.

The discrepancy between the observational error box and the evolution tracks already reduces from $>300 \mathrm{~K}(\mathrm{C} 12)$ to the order of $100 \mathrm{~K}$ (considering the uncertainties) using the new constraints. However, assuming these models to be correct, we would still require $L_{\star} \sim 550 \mathcal{L}_{\odot}$, assuming the $T_{\text {eff }}$ is correct. Given that we have two independent measures of the distance that suggest similar luminosities, it is likely that it is the stellar models that need to be adjusted, based on the assumed $A_{V}=0.01 \mathrm{mag}$ (and therefore $T_{\text {eff }}$ ).

We investigated the effect of assuming non-neglible interstellar extinction ${ }^{5}$ of $A_{V}=0.08$ mag by considering the maximum values from Lallement et al. (2014), although most studies indicate that this should not be the case. This would increase $F_{\text {bol }}$ and consequently increase $T_{\text {eff }}$, for a fixed $\theta$. The increase in the median values of $T_{\text {eff }}$ and $L_{\star}$ as a result of imposing $A_{V}=0.08 \mathrm{mag}$ is indicated by the red vector in Fig. 5 for K18. This could explain some of the discrepancy between our results and evolution models, although such a strong absorption seems unlikely and would also bring the various $T_{\text {eff }}$ determinations into disagreement.

In this work we have assumed a conservative mass of $0.85 \pm$ $0.05 \mathcal{M}_{\odot}$. However, using stellar models we can further constrain the mass by assuming a limited age range to be consistent with a Pop II star. We performed computations of evolutionary tracks with the CESAM2k code (Morel 1997) assuming $[\mathrm{Z} / \mathrm{X}]=-2.4$ which includes an assumed $[\alpha / \mathrm{Fe}] \sim+0.25$, similar to those described in $\mathrm{C} 12$. In order to match the constraints, we are required to lower the mixing-length parameter by $\sim 0.35$ compared to the solar one, to $1.55 \pm 0.03$. Only models with masses in the range of $0.85-0.87 \mathcal{M}_{\odot}$ reach the $L_{\star}$ at an age between 10 and 12 Gyr. A new representative stellar model $\left(0.86 \mathcal{M}_{\odot}, \alpha=1.55\right)$ calculated from CESAM2k is shown in grey in Fig. 5.

\section{Discussion}

We determined an asteroseismic $\log g$ value of $1.39 \pm 0.01$ and $1.42 \pm 0.01$ by using the $v_{\max }$ seismic scaling relation without and with corrections for the mean molecular weight and adiabatic exponent. These values are in statistical agreement with those derived using a parallax from GDR2 (1.43 \pm 0.03). A recent determination from the APOGEE survey (DR15; Majewski et al. 2017) yields a calibrated ${ }^{6} \log g$ of 1.43 dex. These agreements imply that the seismic scaling relation for

\footnotetext{
5 http://stilism.obspm.fr/reddening?frame=icrs\&vlong= 210.63268943\&ulong=deg\&vlat $=09.68609665 \&$ ulat $=$ deg\&valid=

6 The APOGEE stellar parameters provide calibrated and uncalibrated stellar parameters, where the calibration is done independently of the other stellar parameter. For surface gravity, the calibrated value is obtained by adding a constant that is derived from seismic calibrations of spectra. For $T_{\text {eff }}$ however this is obtained by adding a constant derived from photometrical calibrations with models. For the $T_{\text {eff }}$, the calibrated value is not valid in our case.
} 
surface gravity even without corrections is valid in the evolved, metal-poor regime. Our work also validates the use of the corrective terms, although some discrepancy still remains. The consequence of this is important: Today we have access to thousands of giant stars which show oscillation signatures such as $v_{\max }$, and consequently $\log g$ is known with high precision. Fixing this parameter in spectroscopic analyses allows more precise determinations of other spectroscopic parameters and abundances, and can help to uncover systematic errors associated with these analyses.

A recent analysis of the CNO abundances of HD 122563 using 3D hydrodynamical atmospheres by Collet et al. (2018) finds that their imposed surface gravity of $1.61 \pm 0.07$ resulted in discrepant oxygen abundances between molecular and atomic species. They suggest that a downward revision of the order of 0.3 dex would relieve this tension. In this work, the surface gravity has been revised downward by 0.2 dex, close to that proposed by the authors.

Using stellar models and the constraints from the revised luminosity, we refined the mass to within 0.85 and $0.87 \mathcal{M}_{\odot}$. Nevertheless, we still find differences compared to standard stellar models. We looked into the possibility of having a possible increase in interstellar extinction. This would bring the error box in the HR diagram closer to the BASTI stellar evolution tracks (red vector in Fig. 5), because the $T_{\text {eff }}$ would increase. However, the different methodologies (spectroscopic, interferometric, IRFM) would then no longer be in agreement. A more recent measurement of the $T_{\text {eff }}$ with APOGEE spectra yields an uncalibrated (i.e. derived from the spectra) $T_{\text {eff }}$ of $4594 \mathrm{~K}$, also in agreement with the literature $T_{\text {eff }}$ presented here. An alternative is to increase the $L_{\star}$ only, that is, a star that is much further away. From this work we have two independent determinations of the distance yielding similar results. This would also be unlikely. This leaves the only possibility to investigate the stellar models.

In order to match the observational constraints with stellar models, we could increase the metallicity by about 1.0 dex, however, this has been measured by many different authors and this is a very unlikely solution. We could also decrease the initial helium abundance to an extremely low value, but this would be inconsistent with predictions of the primordial abundances, for example Tytler et al. (2000). One of the solutions of this discrepancy is to lower the mixing-length parameter $\alpha$. This decreases the $T_{\text {eff }}$, without influencing the $L_{\star}$. We estimated a shift of $\alpha$ of -0.35 compared to the solar-calibrated one by calculating refined stellar models. Such a value is in agreement with estimations from 3D simulations. Magic et al. 2015 suggest a reduction of $\sim 0.2$ compared to the solar value for a star of this $g,[\mathrm{M} / \mathrm{H}]$, and $T_{\text {eff }}$ (their Fig. 3), where the reduction is primarily due to the lower value of $\log g$.

More recently, Tayar et al. (2017), Viani et al. (2018) and Creevey et al. (2017) investigated the mixing-length parameter empirically by studying samples of stars. For the second two, their analysis concentrated on main sequence stars and subgiants, with the lowest metallicity values close to $-0.60 \mathrm{dex}$, so their results are not applicable to HD 122563. However, they do find a relation that depends on $\log g, T_{\text {eff }}$ and metallicity. Tayar et al. (2017) also restricted their studies to stars with $[\mathrm{Fe} / \mathrm{H}]>-1.0 \mathrm{dex}$, but looked at the specific case of a sample of 3000 giants, with the aim of studying the metallicity dependence. They found that a correction to $\alpha$ of the order of 0.2 per dex is needed. If we apply this correction, we would require a reduction of $\sim 0.5$ dex in $\alpha$ compared to the solar one, not far from what we find. Both empirical and model results indeed support that $\alpha$ needs to be modified in the 1D stellar models. This then would support our new parameter determinations, which consequently validates the seismic scaling relation for $\log g$ in the metal-poor giant regime.

\section{Conclusions}

In this work we described the first detection of oscillations in the metal-poor giant HD 122563. We determined its surface gravity using the detected $v_{\max }$ along with scaling relations. By comparing with the value derived using a Gaia parallax we validated the classical $\left(f_{v_{\max }}=1.0\right)$ seismic scaling relation for $\log g$ in such a metal-poor and non-solar regime. We find a non-significant difference of 0.04 dex. While these relations are valid, applying the corrections for mean molecular weight and the adiabatic exponent results in a smaller discrepancy with the surface gravity derived from the Gaia parallax (0.02 dex).

We have derived updated surface gravity, radius, and luminosity values for HD 122563. These new parameters are quite different from previous determinations. These permit us to make a new estimate of the mass by using stellar models. Only models with masses between 0.85 and $0.87 \mathcal{M}_{\odot}$ satisfy the new constraints. The updated luminosity value along with the literature $T_{\text {eff }}$ provide a new error box in the HR diagram. The large difference on the $T_{\text {eff-axis found in earlier works has significantly }}$ reduced, to the order of $100 \mathrm{~K}$. This final difference can be rectified by modifying the mixing-length parameter used in the models. Our model required a change of -0.35 compared to the solar-calibrated value, a value in agreement with $3 \mathrm{D}$ simulations and empirically derived values.

SONG radial velocity observations are continuing in order to determine $\langle\Delta v\rangle$ and resolve the individual frequencies. These observations will help to test scaling relations for the radius and the mass using $\langle\Delta v\rangle$ outside of the solar regime, and bring important constraints on the knowledge of the fundamental parameters of this star including its age. Complementing these data with high precision parallax measurements will allow us to derive accurate masses and radii independent of models.

Acknowledgements. We are grateful for the Programme National de Physique Stellaire for financial support for this research project. This work is based on observations made with the Hertzsprung SONG telescope operated on the Spanish Observatorio del Teide on the island of Tenerife by the Aarhus and Copenhagen Universities and by the Instituto de Astrofisica de Canarias. Support for the construction of the Hertzsprung SONG Telescope from the Instituto de Astrofisica de Canarias, the Villum Foundation, the Carlsberg Foundation and the Independent Research Fund Denmark is gratefully acknowledged. E.C. is funded by the European Union's Horizon 2020 research and innovation programme under the Marie Sklodowska-Curie grant agreement No. 664931. D.S. acknowledges the financial support from the CNES GOLF grant and the Observatoire de la Côte d'Azur for support during his stays. We also deeply acknowledge the Gaia team for their huge efforts in bringing us high quality data.

\section{References}

Amarsi, A. M., Nordlander, T., Barklem, P. S., et al. 2018, A\&A, 615, A139 Andersen, M. F., Grundahl, F., Christensen-Dalsgaard, J., et al. 2014, Rev. Mex. Astron. Astrofis. Conf. Ser., 45, 83

Arentoft, T., Kjeldsen, H., Nuspl, J., et al. 1998, A\&A, 338, 909

Bedding, T. R., Kjeldsen, H., Butler, R. P., et al. 2004, ApJ, 614, 380

Belkacem, K., Goupil, M. J., Dupret, M. A., et al. 2011, A\&A, 530, A142

Bonaca, A., Tanner, J. D., Basu, S., et al. 2012, ApJ, 755, L12

Brogaard, K., Hansen, C. J., Miglio, A., et al. 2018, MNRAS, 476, 3729

Brown, T. M., \& Gilliland, R. L. 1994, ARA\&A, 32, 37

Butler, R. P., Bedding, T. R., Kjeldsen, H., et al. 2004, ApJ, 600, L75

Casagrande, L., Portinari, L., Glass, I. S., et al. 2014, MNRAS, 439, 2060

Chaplin, W. J., Kjeldsen, H., Christensen-Dalsgaard, J., et al. 2011, Science, 332, 213

Coelho, H. R., Chaplin, W. J., Basu, S., et al. 2015, MNRAS, 451, 3011 
Collet, R., Asplund, M., \& Thévenin, F. 2005, A\&A, 442, 643

Collet, R., Nordlund, Ã., Asplund, M., Hayek, W., \& Trampedach, R. 2018, MNRAS, 475, 3369

Corsaro, E., \& De Ridder, J. 2014, A\&A, 571, A71

Corsaro, E., Grundahl, F., Leccia, S., et al. 2012, A\&A, 537, A9

Corsaro, E., De Ridder, J., \& García, R. A. 2015, A\&A, 579, A83

Creevey, O. L., Doğan, G., Frasca, A., et al. 2012a, A\&A, 537, A111

Creevey, O. L., Thévenin, F., Boyajian, T. S., et al. 2012b, A\&A, 545, A17

Creevey, O. L., Thévenin, F., Basu, S., et al. 2013, MNRAS, 431, 2419

Creevey, O. L., Metcalfe, T. S., Schultheis, M., et al. 2017, A\&A, 601, A67

Epstein, C. R., Elsworth, Y. P., Johnson, J. A., et al. 2014, ApJ, 785, L28

Frandsen, S., Jones, A., Kjeldsen, H., et al. 1995, A\&A, 301, 123

Gaia Collaboration (Brown, A. G. A., et al.) 2018, A\&A, 616, A1

Gilmore, G., Randich, S., Asplund, M., et al. 2012, The Messenger, 147, 25

Grundahl, F., Fredslund Andersen, M., Christensen-Dalsgaard, J., et al. 2017, ApJ, 836, 142

Heiter, U., Jofré, P., Gustafsson, B., et al. 2015, A\&A, 582, A49

Hekker, S., Elsworth, Y., Mosser, B., et al. 2013, A\&A, 556, A59

Houdashelt, M. L., Bell, R. A., \& Sweigart, A. V. 2000, AJ, 119, 1448

Jofré, P., Heiter, U., Worley, C. C., et al. 2017, A\&A, 601, A38

Joyce, M., \& Chaboyer, B. 2018, ApJ, 856, 10

Kallinger, T., Beck, P. G., Stello, D., \& Garcia, R. A. 2018, A\&A, 616, A104

Karovicova, I., White, T. R., Nordlander, T., et al. 2018, MNRAS, 475, L81

Kjeldsen, H., \& Bedding, T. R. 1995, A\&A, 293, 87

Kjeldsen, H., Bedding, T. R., Butler, R. P., et al. 2005, ApJ, 635, 1281

Lallement, R., Vergely, J.-L., Valette, B., et al. 2014, A\&A, 561, A91

Lebreton, Y., Perrin, M.-N., Cayrel, R., Baglin, A., \& Fernandes, J. 1999, A\&A, 350,587

Lindegren, L., Hernández, J., Bombrun, A., et al. 2018, A\&A, 616, A2

Luri, X., Brown, A. G. A., Sarro, L. M., et al. 2018, A\&A, 616, A9

Magic, Z., Weiss, A., \& Asplund, M. 2015, A\&A, 573, A89

Majewski, S. R., Schiavon, R. P., Frinchaboy, P. M., et al. 2017, AJ, 154, 94

Morel, P. 1997, A\&AS, 124, 597

Morel, T., \& Miglio, A. 2012, MNRAS, 419, L34

Pietrinferni, A., Cassisi, S., Salaris, M., \& Castelli, F. 2004, ApJ, 612, 168

Prakapavičius, D., Kučinskas, A., Dobrovolskas, V., et al. 2017, A\&A, 599, A128

Prša, A., Harmanec, P., Torres, G., et al. 2016, AJ, 152, 41

Sharma, S., Stello, D., Bland-Hawthorn, J., Huber, D., \& Bedding, T. R. 2016, ApJ, 822, 15
Tayar, J., Somers, G., Pinsonneault, M. H., et al. 2017, ApJ, 840, 17

Thévenin, F., \& Idiart, T. P. 1999, ApJ, 521, 753

Trampedach, R., Aarslev, M. J., Houdek, G., et al. 2017, MNRAS, 466, L43

Tytler, D., O'Meara, J. M., Suzuki, N., \& Lubin, D. 2000, Phys. Scr. Vol. T, 85, 12

van Leeuwen, F. 2007, A\&A, 474, 653

Viani, L. S., Basu, S., Chaplin, W. J., Davies, G. R., \& Elsworth, Y. 2017, ApJ, 843,11

Viani, L. S., Basu, S., Joel Ong, J. M., Bonaca, A., \& Chaplin, W. J. 2018, ApJ, 858,28

Yu, J., Huber, D., Bedding, T. R., et al. 2018, ApJS, 236, 42

\section{Appendix A: Software and observations}

This work made use of the following free public data and software

- The radial velocity data were obtained using the Hertzsprung SONG telescope, which is operated on the Spanish Observatorio del Teide on the island of Tenerife by the Aarhus and Copenhagen Universities and by the Instituto de Astrofisica de Canarias.

- Parallaxes from the ESA Gaia Space Mission Data Release 2.

- The VizieR catalogue access tool, CDS, Strasbourg, France. The original description of the VizieR service was published in A\&AS, 143, 23.

- The radial velocities were analysed using the SONG reduction pipeline.

- Extinction map tools from the Stilism project https:// stilism.obspm.fr/

- The frequency analysis was done using the Diamonds code. This code is available at https://github.com/ EnricoCorsaro/DIAMONDS.

- This article was prepared using overleaf.

- The figures were prepared using Jupyter-Notebook. 